\title{
Kidney function and cognitive decline in an oldest-old Chinese population
}

This article was published in the following Dove Press journal:

Clinical Interventions in Aging

4 July 2017

Number of times this article has been viewed

\section{Kunhao Bai ${ }^{1,2, *}$ \\ Yujing $\operatorname{Pan}^{3, *}$ \\ Fanghong $\mathrm{Lu}^{4}$ \\ Yingxin Zhao ${ }^{4}$ \\ Jinwei Wang ${ }^{1,2}$ \\ Luxia Zhang ${ }^{1,2}$}

'Renal Division, Department of Medicine, Peking University First Hospital, Beijing, People's Republic of China; ${ }^{2}$ Peking University Institute of Nephrology, Peking University Health Science Center, Beijing, People's Republic of China; ${ }^{3}$ Renal Division, Department of Medicine, Peking University International Hospital, Beijing, People's Republic of China; ${ }^{4}$ Cardio-Cerebrovascular Control and Research Center, Institute of Basic Medicine, Shandong Academy of Medical Sciences, Jinan, Shandong, People's Republic of China

*These authors contributed equally to this work
Correspondence: Jinwei Wang

Renal Division, Department of Medicine, Peking University First Hospital, 8 Xishiku Street, Xicheng District, Beijing 100034 , People's Republic of China

Tel +86 I0 83572388

Fax +86 $106655 \quad 1055$

Email wangjinwei@bjmu.edu.cn
Background: Early-stage chronic kidney disease has been suggested to be correlated with cognitive decline, but the association has rarely been explored in the oldest old.

Subjects and methods: This prospective study included 284 Chinese participants aged 80 years or older with serum creatinine levels $<150 \mu \mathrm{mol} / \mathrm{L}$. The median follow-up time was 3.3 years, and 247 (87.0\%) participants provided valid data at their last visit. Kidney function was evaluated by measuring the estimated glomerular filtration rate (eGFR) at baseline, and cognitive function was evaluated using the Mini-Mental State Examination (MMSE) at both baseline and annual visits. A reliable decrease in the MMSE score over the follow-up period was observed based on a Reliable Change Index of 1.645 (equivalent to a 90\% confidence interval $[\mathrm{CI}]$ ), which was used to define cognitive decline. Poisson regression models were built to analyze the association between baseline kidney function and cognitive decline.

Results: A total of $18(7.3 \%)$ cases of incident cognitive decline were observed during the follow-up period. After adjusting for potential confounders, the relative risk of developing cognitive decline was 4.03 (95\% CI 1.09-13.81) among participants with an eGFR of $30-59 \mathrm{~mL} / \mathrm{min} / 1.73 \mathrm{~m}^{2}$ compared to participants with an eGFR of $\geq 60 \mathrm{~mL} / \mathrm{min} / 1.73 \mathrm{~m}^{2}$.

Conclusion: Early-stage chronic kidney disease was correlated with cognitive decline in an oldest-old Chinese population.

Keywords: chronic kidney disease, cognitive decline, oldest old

\section{Introduction}

The prevalence of chronic kidney disease (CKD) increases markedly with age, ${ }^{1-3}$ with the highest prevalence observed among the oldest-old people, ie, those aged 80 years or older. ${ }^{3,4}$ The prevalence of cognitive decline including dementia also increases with age ${ }^{5}$ with an estimated prevalence of approximately $20 \%$ in individuals aged 80 years. ${ }^{6}$ Both CKD and cognitive decline are major public health problems in aging populations and pose an enormous societal burden. ${ }^{7}$

As shown in several studies conducted in the past decade, kidney function is associated with cognitive decline.$^{8-11}$ However, to the best of our knowledge, no longitudinal study has investigated this association in an oldest-old Asian population.

Therefore, we prospectively investigated the association between kidney function and cognitive decline in an oldest-old Chinese population ( $\geq 80$ years) with serum creatinine levels $<150 \mu \mathrm{mol} / \mathrm{L}$.

\section{Subjects and methods}

\section{Subjects}

In 2004, 284 participants aged 80 years or older from Shandong, People's Republic of China, were recruited to participate in a double-blind placebo-controlled trial designed to 
establish the benefits and risks of antihypertensive treatment; this trial included an assessment of cognitive function. ${ }^{12}$ All participants had hypertension (systolic pressure 160$200 \mathrm{mmHg}$; diastolic pressure $<110 \mathrm{mmHg}$ ), serum creatinine levels $<150 \mu \mathrm{mol} / \mathrm{L}$, and no clinical diagnosis of dementia at baseline. Cognitive function was assessed with the Mini-Mental State Examination (MMSE) at both the baseline and annual visits. Participants were randomly assigned to receive antihypertensive treatment or placebo. After the trial ended in 2007, all participants received antihypertensive treatment and were followed for 1 year. Full details of the protocol have been published elsewhere. ${ }^{13}$ In total, 247 participants (87.0\%) provided valid MMSE data at their last visit. Written informed consent was obtained from all study participants, and the trial was approved by the ethics committee of Beijing Hypertension League Institute.

\section{Evaluation of cognitive function}

All participants were assessed with the MMSE at baseline and annual visits. The MMSE is a brief standardized instrument that has been extensively used to assess individuals' cognitive function. ${ }^{14}$ The MMSE score ranges from 0 to 30, with higher scores indicating better cognitive function. The MMSE assesses attention, orientation, language, ability to follow simple verbal and written commands, and immediate and short-term recall. The scores were calculated by the coordinating office staff. ${ }^{13}$

According to previous studies, not all changes in the MMSE score reflect true clinical cognitive decline or improvement. Changes in MMSE test scores may also result from a practice effect, measurement error, regression to the mean, or normal aging. ${ }^{15}$ The Reliable Change Index (RCI) is a statistical method that estimates the probability that the change in an individual's test scores is not due to chance. The RCI generated by Chelune et $\mathrm{al}^{16}$ accounts for practice effects and measurement error, whereas $\mathrm{Hsu}^{17}$ proposed an RCI that also accounts for regression to the mean. Both equations have been described in detail elsewhere. ${ }^{16,17}$ We calculated the RCI using both methods to improve the accuracy of the definition of cognitive decline. Based on a previous study of an elderly Chinese population, the test-retest reliability that we used for the calculation was $0.75 .{ }^{18}$ A reliable decrease in the MMSE score was defined by an RCI of 1.645 (equivalent to a $90 \%$ confidence interval [CI]).

\section{Evaluation of renal function at baseline}

Baseline serum creatinine levels of all participants were measured by Jaffe's kinetic method using a Hitachi 7600 automated biochemical analyzer (Hitachi, Tokyo, Japan). The estimated glomerular filtration rate (eGFR) was calculated using the modified modification of diet in renal disease (MDRD) equation, which was developed using data from Chinese participants with CKD. ${ }^{19}$ All participants had an eGFR $>30 \mathrm{~mL} / \mathrm{min} / 1.73 \mathrm{~m}^{2}$. Early-stage CKD was defined as an eGFR of $30-59 \mathrm{~mL} / \mathrm{min} / 1.73 \mathrm{~m}^{2}$.

\section{Evaluation of covariates at baseline}

A detailed description of this procedure has been published elsewhere. ${ }^{13}$ Baseline blood pressure measurements were collected from all participants. A questionnaire was used to obtain information on previous cardiovascular diseases (including myocardial infarction, atrial fibrillation, and stroke) and lifestyle behaviors. Body mass index was calculated as weight $(\mathrm{kg})$ divided by height squared $\left(\mathrm{m}^{2}\right)$. Hemoglobin, fasting glucose, uric acid, and total cholesterol levels were measured at baseline.

\section{Statistical analysis}

Standard descriptive statistics were used to assess the baseline characteristics and to test for differences in the characteristics between participants with and without cognitive decline. Continuous variables were presented as mean \pm SD or as the median (interquartile range $[\mathrm{IQR}]$ ), and binary variables were presented as proportions. Data were compared using an independent $t$-test for continuous variables and $\chi^{2}$ test or Fisher's exact test for binary variables.

Poisson regression models were built to analyze the association between baseline kidney function and cognitive decline (presented as the relative risk [RR] and 95\% $\mathrm{CI})$. We first analyzed the eGFR as a binary variable: $\geq 60$ (reference) and $30-59 \mathrm{~mL} / \mathrm{min} / 1.73 \mathrm{~m}^{2}$. Multivariate models were then constructed to adjust for potential confounding variables associated with kidney function and/ or cognitive function, including age (continuous), gender (male vs female), body mass index (continuous), current smoking (no vs yes), previous cardiovascular disease (no vs yes), diastolic blood pressure (continuous), fasting blood glucose level (continuous), and total cholesterol level (continuous). Crude and adjusted RRs with 95\% CIs are presented. We also used the same strategy to analyze eGFR with three categories: $\geq 60 \mathrm{~mL} / \mathrm{min} / 1.73 \mathrm{~m}^{2}$ (reference), $45-59 \mathrm{~mL} / \mathrm{min} / 1.73 \mathrm{~m}^{2}$, and $30-44 \mathrm{~mL} / \mathrm{min} / 1.73 \mathrm{~m}^{2}$.

We then analyzed the eGFR as a continuous variable (per $10 \mathrm{~mL} / \mathrm{min} / 1.73 \mathrm{~m}^{2}$ increase). A similar Poisson regression model was then generated. Moreover, as the accuracy of the eGFR calculated using equations to evaluate kidney function in the oldest old is controversial, we also used serum creatinine levels (continuous, per $0.1 \mathrm{mg} / \mathrm{dL}$ increase) to analyze the association using the same methods. 
All $P$-values were two-tailed. Statistical tests were performed using SAS software version 9.4 (SAS Institute Inc., Cary, NC, USA).

\section{Results}

A total of 247 participants (87.0\%) provided valid MMSE data at their last visit. The median and IQR of these participants' MMSE scores at baseline was 22 (20-23), and the change in MMSE score during follow-up was $2(1-4)$. The distribution of responders and nonresponders was comparable in age ( $83.5 \pm 2.6$ vs $83.3 \pm 2.7, P=0.76)$, gender (percentage of males $25.2 \%$ vs $32.4 \%, P=0.35$ ), percentage with previous cardiovascular disease ( $5.3 \%$ vs $2.7 \%, P=1.0$ ), and treatment group $(53.7 \%$ vs $38.9 \%, P=0.23)$, but not the percentage of current smokers ( $6.1 \%$ vs $18.9 \%, P=0.01)$.

The baseline characteristics of the responders are shown in Table 1. The average age was $83.5 \pm 2.6$ years, and $25.2 \%$ were male. Compared to subjects without cognitive decline, participants who developed cognitive decline were more likely to be male and had lower fasting blood glucose levels, higher diastolic blood pressure, higher serum creatinine levels, and a lower eGFR. The number of participants with eGFR $\geq 60$ and $30-59 \mathrm{~mL} / \mathrm{min} / 1.73 \mathrm{~m}^{2}$ was 110 and 137 , respectively.

A total of 18 (7.3\%) cases of incident cognitive decline were observed during the follow-up period. In the crude analysis (Table 2), the RR of cognitive decline among participants with an eGFR of $30-59 \mathrm{~mL} / \mathrm{min} / 1.73 \mathrm{~m}^{2}$ was $4.01(95 \%$ CI 1.16-13.87). After adjusting for potential confounding variables, including age, gender, body mass index, current smoking, previous cardiovascular disease, fasting blood glucose levels, diastolic blood pressure (baseline diastolic blood pressure was associated with incident dementia in a previous study $^{20}$ ), and total cholesterol levels, multivariate-adjusted RR of cognitive decline among participants with an eGFR of 30-59 mL/min $/ 1.73 \mathrm{~m}^{2}$ was 4.03 (95\% CI 1.09-14.90).

When the eGFR was analyzed as a three-category variable (Table 3), the number of participants with eGFR $\geq 60,45-59$, and $30-44 \mathrm{~mL} / \mathrm{min} / 1.73 \mathrm{~m}^{2}$ was 110,82 , and 55 , respectively. The multivariate-adjusted RR among participants with an eGFR of $45-59$ and $30-44 \mathrm{~mL} / \mathrm{min} / 1.73 \mathrm{~m}^{2}$ was $2.75(95 \%$ CI 0.65-11.67) and 7.29 (1.66-31.90), respectively.

When the eGFR was analyzed as a continuous variable (Table 4), the multivariate-adjusted RR for cognitive decline per $10 \mathrm{~mL} / \mathrm{min} / 1.73 \mathrm{~m}^{2}$ increase in eGFR was 0.68 (95\% CI $0.47-0.97)$. When the serum creatinine level was used to evaluate kidney function, the multivariate-adjusted RR for cognitive decline per $0.1 \mathrm{mg} / \mathrm{dL}$ increase in serum creatinine level was 1.34 (95\% CI 1.08-1.68).

\section{Discussion}

In our previous study, which included a community-based population of 1,351 Chinese individuals $>40$ years old with an eGFR of $>30 \mathrm{~mL} / \mathrm{min} / 1.73 \mathrm{~m}^{2}$, the odds ratio of

Table I Baseline characteristics of the participants according to cognitive decline status

\begin{tabular}{|c|c|c|c|c|}
\hline Variables & All participants & $\begin{array}{l}\text { Participants without } \\
\text { cognitive decline }\end{array}$ & $\begin{array}{l}\text { Participants with } \\
\text { cognitive decline }\end{array}$ & $P$-value \\
\hline Number of participants & 247 & 229 & 18 & \\
\hline Male, \% & 25.2 & 23.7 & 44.4 & 0.09 \\
\hline Age, years & $83.5 \pm 2.6$ & $83.5 \pm 2.7$ & $82.9 \pm 2.2$ & 0.36 \\
\hline Current smoking, \% & 6.1 & 5.7 & II.I & 0.30 \\
\hline Previous cardiovascular disease, \% & 5.3 & 5.2 & 5.6 & 1.00 \\
\hline Treatment group, \% & 52.6 & 53.7 & 38.9 & 0.23 \\
\hline Body mass index & $23.8 \pm 14.2$ & $23.9 \pm 14.7$ & $22.6 \pm 1.6$ & 0.25 \\
\hline Hemoglobin, g/dL & $12.2 \pm 1.4$ & $12.2 \pm 1.4$ & $11.9 \pm 1.6$ & 0.49 \\
\hline Fasting blood glucose, $\mathrm{mmol} / \mathrm{L}$ & $5.0 \pm 0.8$ & $5.0 \pm 0.8$ & $4.6 \pm 0.6$ & 0.05 \\
\hline Total cholesterol, mmol/L & $4.6 \pm 0.8$ & $4.6 \pm 0.8$ & $4.6 \pm 0.6$ & 0.87 \\
\hline Serum uric acid, $\mu \mathrm{mol} / \mathrm{L}$ & $258.8 \pm 78.8$ & $257.0 \pm 77.9$ & $282.4 \pm 88.2$ & 0.19 \\
\hline Systolic BP, mmHg & $176.4 \pm 13.1$ & $176.5 \pm 13.2$ & $|78.0 \pm| \mid .3$ & 0.64 \\
\hline Diastolic BP, mmHg & $84.3 \pm 9.9$ & $83.9 \pm 9.9$ & $89.8 \pm 7.4$ & 0.01 \\
\hline Serum creatinine, $\mu \mathrm{mol} / \mathrm{L}$ & $100.0 \pm 23.9$ & $98.6 \pm 23.5$ & || $8.5 \pm 2 \mid .7$ & 0.001 \\
\hline eGFR, $\mathrm{mL} / \mathrm{min} / \mathrm{I} .73 \mathrm{~m}^{2}$ & $62.6 \pm 22.1$ & $63.5 \pm 22.2$ & $51.9 \pm 18.7$ & 0.03 \\
\hline \multicolumn{5}{|l|}{ Categories of eGFR, \% } \\
\hline$\geq 60 \mathrm{~mL} / \mathrm{min} / \mathrm{I} .73 \mathrm{~m}^{2}$ & 44.5 & 46.7 & 16.7 & 0.01 \\
\hline $30-59 \mathrm{~mL} / \mathrm{min} / \mathrm{I} .73 \mathrm{~m}^{2}$ & 55.5 & 53.3 & 83.3 & 0.01 \\
\hline Median MMSE score (IQR) & $22(20-23)$ & $21(20-23)$ & $25(23-29)$ & $<0.001$ \\
\hline
\end{tabular}

Note: Data are presented as percentages or mean \pm SD unless indicated otherwise.

Abbreviations: BP, blood pressure; eGFR, estimated glomerular filtration rate; IQR, interquartile range; MMSE, Mini-Mental State Examination. 
Table 2 Kidney function and cognitive decline

\begin{tabular}{|c|c|c|}
\hline $\begin{array}{l}\text { eGFR and cognitive } \\
\text { decline }\end{array}$ & $\begin{array}{l}\text { eGFR } \geq 60 \\
(n=I I 0)\end{array}$ & $\begin{array}{l}\text { eGFR 30-59 } \\
(n=137)\end{array}$ \\
\hline Crude RR & Reference & $4.01(1.16-13.87)$ \\
\hline Age, gender-adjusted RR & Reference & $5.16(1.46-18.25)$ \\
\hline Multivariable-adjusted $\mathrm{RR}^{\mathrm{a}}$ & Reference & $4.03(1.09-14.90)$ \\
\hline
\end{tabular}

Notes: Data are presented as RR $(95 \% \mathrm{Cl})$. ${ }^{\mathrm{a}}$ The model was further adjusted for body mass index, current smoking status, previous cardiovascular disease, fasting blood glucose level, diastolic BP, total cholesterol level, and treatment group.

Abbreviations: BP, blood pressure; eGFR, estimated glomerular filtration rate $\left(\mathrm{mL} / \mathrm{min} / \mathrm{l} .73 \mathrm{~m}^{2}\right)$; RR, relative risk.

developing cognitive decline was 2.73 (95\% CI 1.00-7.56) among participants with an eGFR of $30-59 \mathrm{~mL} / \mathrm{min} / 1.73 \mathrm{~m}^{2}$ compared to those with an eGFR of $>90 \mathrm{~mL} / \mathrm{min} / 1.73 \mathrm{~m}^{2} .{ }^{21}$ Based on the current prospective study, early-stage CKD (an eGFR of $30-59 \mathrm{~mL} / \mathrm{min} / 1.73 \mathrm{~m}^{2}$ ) was also associated with an increased risk of cognitive decline in an oldest-old Chinese population; this association was independent of age, gender, and other confounders. Given the high prevalence of early-stage CKD in the oldest old, ${ }^{4} \mathrm{CKD}$ poses substantial risk as a contributing factor to cognitive decline.

The association between CKD and cognitive function has been recognized in recent decades. According to several cross-sectional and longitudinal studies, CKD is associated with an increased risk of cognitive decline in adults. In a study by Kurella et al, ${ }^{22} \mathrm{CKD}$ was associated with an increased risk of cognitive impairment in the elderly, and this risk could not be fully explained by other well-established risk factors; furthermore, more advanced stages of CKD were found to be associated with an increased risk of cognitive impairment. Khatri et $\mathrm{al}^{23}$ reported an association between decreased kidney function and cognitive decline in individuals with even mild CKD. In addition, Buchman et $\mathrm{al}^{8}$ observed an association between impaired kidney function and a more rapid rate of cognitive decline in the elderly. However, Slinin et $\mathrm{al}^{24}$ did not observe an independent association of mildto-moderate reductions in kidney function with increased

Table 3 eGFR and cognitive decline

\begin{tabular}{llll}
\hline $\begin{array}{l}\text { eGFR and } \\
\text { cognitive decline }\end{array}$ & $\begin{array}{l}\text { eGFR } \geq 60 \\
(\mathbf{n}=|\mathrm{I}| \mathbf{0})\end{array}$ & $\begin{array}{l}\text { eGFR 45-59 } \\
(\mathbf{n = 8 2})\end{array}$ & $\begin{array}{l}\text { eGFR 30-44 } \\
(\mathbf{n}=55)\end{array}$ \\
\hline $\begin{array}{l}\text { Crude RR } \\
\begin{array}{l}\text { Age, gender- } \\
\text { adjusted RR }\end{array}\end{array}$ & Reference & $3.13(0.8 I-12.10)$ & $5.33(\mathrm{I} .4 \mathrm{I}-20.10)$ \\
$\begin{array}{l}\text { Multivariable- } \\
\text { adjusted RR }\end{array}$ & Reference & $3.82(0.97-15.00)$ & $7.9 I(2.0 \mathrm{I}-3 \mathrm{I} .10)$ \\
\hline
\end{tabular}

Notes: Data are presented as RR $(95 \% \mathrm{Cl}) .{ }^{\text {a The model was further adjusted for }}$ body mass index, current smoking status, previous cardiovascular disease, fasting blood glucose level, diastolic BP, total cholesterol level, and treatment group.

Abbreviations: BP, blood pressure; eGFR, estimated glomerular filtration rate $\left(\mathrm{mL} / \mathrm{min} / \mathrm{l} .73 \mathrm{~m}^{2}\right) ; R R$, relative risk.
Table 4 eGFR, serum creatinine, and cognitive decline

\begin{tabular}{lll}
\hline & $\begin{array}{l}\text { eGFR, per } \\
10 \mathrm{~mL} / \mathrm{min} / 1.73 \mathrm{~m}^{2} \\
\text { increase }\end{array}$ & $\begin{array}{l}\text { Serum creatinine } \\
\text { per } 0.1 \mathrm{mg} / \mathrm{dL} \\
\text { increase }\end{array}$ \\
\hline Crude RR & $0.72(0.52-0.99)$ & $1.37(1.12-1.67)$ \\
Age, gender-adjusted RR & $0.65(0.45-0.92)$ & $1.34(1.10-1.62)$ \\
Multivariable-adjusted RR & $0.68(0.47-0.97)$ & $1.34(1.08-1.68)$ \\
\hline
\end{tabular}

Notes: Data are presented as RR $(95 \% \mathrm{Cl})$. ${ }^{\text {aThe model was further adjusted for }}$ body mass index, current smoking status, previous cardiovascular disease, fasting blood glucose level, diastolic BP, total cholesterol level, and treatment group.

Abbreviations: BP, blood pressure; eGFR, estimated glomerular filtration rate $\left(\mathrm{mL} / \mathrm{min} / 1.73 \mathrm{~m}^{2}\right) ; R R$, relative risk.

global cognitive impairment or risk of cognitive decline in older men. In addition, Helmer et $\mathrm{a}^{9}{ }^{9}$ did not observe an association between increased risk of cognitive decline or dementia and low eGFR values, but a faster decrease in eGFR and proteinuria were associated with global cognitive decline and incident dementia with a vascular component. A more plausible explanation is that a rapid decrease in eGFR and proteinuria reflect more severe kidney damage or dysfunction, whereas a low eGFR alone is common during aging in the absence of a true pathologic process; these explanations should be verified in further investigations.

Several mechanisms may explain the association between kidney function and cognitive decline. Small vessel disease in the brain and kidney is considered to be a key contributor to this relationship. ${ }^{25}$ Hemodynamic similarities in vascular beds between the brain and the kidney have been observed. ${ }^{25}$ In addition, both the brain and kidney are lowresistance end organs that are exposed to a high volume of blood flow throughout the cardiac cycle..$^{25}$ The exposure of small vessels to high flow and high pulsatile pressure may explain the presence of microvascular damage resulting in cognitive function deterioration and renal insufficiency. ${ }^{26}$ The brain and kidney also share common cardiovascular risk factors, such as hypertension, hypercholesterolemia, diabetes mellitus, and smoking, which entail pathological microvascular changes mediated by oxidative and inflammatory processes occurring in similar endothelial structures and low-resistance vascular beds. ${ }^{27}$ Impaired endothelial function is manifested as susceptibility to white matter changes, lacunar infarcts, microinfarcts, and defects in the blood-brain barrier in the brain and as impaired glomerular filtration and secondary proteinuria in the kidney. ${ }^{27}$ Moreover, anemia ${ }^{28}$ and hyperhomocysteinemia ${ }^{29}$ are common even in earlystage CKD and have been suggested to be correlated with incident dementia.

Our study has several limitations. First, we did not include a large number of participants, and the median follow-up 
period of 3.3 years for repeated cognitive function tests was comparatively short. Both of these limitations resulted in a relatively small number of cases of incident cognitive decline, and this small sample size limited the statistical power of the analyses. Second, we lacked information on the participants' educational background, which is significantly associated with cognitive function. However, the median MMSE score of all participants was 22, which was relatively low, and the IQR of the MMSE score was 20-23, which was relatively narrow. These low scores might have occurred because all participants were recruited from the same region and were aged 80 years or older; we speculate that all participants might have had a similar and relatively insufficient level of education. Third, an optimal method for estimating kidney function among the oldest old has not been clearly established. We used both the eGFR and serum creatinine levels to evaluate kidney function, and the results were consistent, suggesting that the findings presented in this study were robust. Fourth, we used MMSE scores to define cognitive decline instead of a gold standard method involving a psychiatrist-obtained history and interactions with the family and a caregiver. Fifth, albuminuria may be correlated with cognitive decline. However, information regarding the participants' urinalysis was missing. Finally, as our study was observational, residual confounders of some unmeasured covariates may exist.

Despite these limitations, our study had several strengths. First, we explored the relationship between kidney function and cognitive decline in an oldest-old population, and this relationship has only been investigated in a few studies. Second, we calculated the RCI using two methods simultaneously to exclude a practice effect, measurement error, and regression to the mean, and this approach improved the accuracy of the definition of cognitive decline. Third, we used improved statistical methods compared to those applied in previous studies. To analyze the associations, we generated Poisson regression models, which are more suitable when the probability of the outcome is low. ${ }^{30} \mathrm{We}$ also used the Poisson regression models to estimate $\mathrm{RR}$, which is preferred over the odds ratio in most prospective investigations.

\section{Conclusion}

Our prospective study suggests that early-stage CKD is associated with cognitive decline in an oldest-old Chinese population. These findings may help clinicians identify individuals at a high risk of developing cognitive decline, and strategies to prevent or delay the incidence of cognitive decline in the oldest old should thus be targeted in further research.

\section{Acknowledgments}

This study was supported by the Research Special Fund for Public Welfare Industry of Health from the National Health and Family Planning Commission of the People's Republic of China (201002010); the National Key Technology R\&D Program of the Ministry of Science and Technology (2011BAI10B01); and the Establishment of an Early Diagnosis Pathway and Model for Evaluating the Progression of Chronic Kidney Disease (D131100004713007) from the Beijing Science and Technology Committee.

\section{Disclosure}

The authors report no conflicts of interest in this work.

\section{References}

1. Garg AX, Papaioannou A, Ferko N, Campbell G, Clarke JA, Ray JG Estimating the prevalence of renal insufficiency in seniors requiring long-term care. Kidney Int. 2004;65(2):649-653.

2. Zhang QL, Koenig W, Raum E, Stegmaier C, Brenner H, Rothenbacher D. Epidemiology of chronic kidney disease: results from a population of older adults in Germany. Prev Med. 2009;48(2):122-127.

3. Bowling CB, Inker LA, Gutierrez OM, et al. Age-specific associations of reduced estimated glomerular filtration rate with concurrent chronic kidney disease complications. Clin J Am Soc Nephrol. 2011;6(12): 2822-2828.

4. Bowling CB, Muntner P. Epidemiology of chronic kidney disease among older adults: a focus on the oldest old. J Gerontol A Biol Sci Med Sci. 2012;67(12):1379-1386.

5. Graham JE, Rockwood K, Beattie BL, et al. Prevalence and severity of cognitive impairment with and without dementia in an elderly population. Lancet. 1997;349(9068):1793-1796.

6. Lobo A, Launer LJ, Fratiglioni L, et al. Prevalence of dementia and major subtypes in Europe: a collaborative study of population-based cohorts. Neurologic Diseases in the Elderly Research Group. Neurology. 2000;54(11 suppl 5):S4-S9.

7. Murray AM. Cognitive impairment in the aging dialysis and chronic kidney disease populations: an occult burden. Adv Chronic Kidney Dis. 2008;15(2):123-132.

8. Buchman AS, Tanne D, Boyle PA, Shah RC, Leurgans SE, Bennett DA. Kidney function is associated with the rate of cognitive decline in the elderly. Neurology. 2009;73(12):920-927.

9. Helmer C, Stengel B, Metzger M, et al. Chronic kidney disease, cognitive decline, and incident dementia: the 3C Study. Neurology. 2011; 77(23):2043-2051.

10. Feng L, Yap KB, Yeoh LY, Ng TP. Kidney function and cognitive and functional decline in elderly adults: findings from the Singapore longitudinal aging study. J Am Geriatr Soc. 2012;60(7):1208-1214.

11. Etgen T, Sander D, Chonchol M, et al. Chronic kidney disease is associated with incident cognitive impairment in the elderly: the INVADE study. Nephrol Dial Transplant. 2009;24(10):3144-3150.

12. Beckett NS, Peters R, Fletcher AE, et al. Treatment of hypertension in patients 80 years of age or older. $N$ Engl J Med. 2008;358(18): 1887-1898.

13. Bulpitt C, Fletcher A, Beckett N, et al. Hypertension in the Very Elderly Trial (HYVET): protocol for the main trial. Drugs Aging. 2001;18(3): 151-164.

14. Folstein MF, Folstein SE, McHugh PR. "Mini-mental state". A practical method for grading the cognitive state of patients for the clinician. J Psychiatr Res. 1975;12(3):189-198.

15. Hensel A, Angermeyer MC, Riedel-Heller SG. Measuring cognitive change in older adults: reliable change indices for the Mini-Mental State Examination. J Neurol Neurosurg Psychiatry. 2007;78(12):1298-1303. 
16. Chelune GJ, Naugle RI, Lüders H, Sedlak J, Awad IA. Individual change after epilepsy surgery: practice effects and base-rate information. Neuropsychology. 1993;7(1):41-52.

17. Hsu LM. Caveats concerning comparisons of change rates obtained with five methods of identifying significant client changes: comment on Speer and Greenbaum (1995). J Consult Clin Psychol. 1999;67(4): 594-598.

18. Xu G, Meyer JS, Huang Y, Du F, Chowdhury M, Quach M. Adapting mini-mental state examination for dementia screening among illiterate or minimally educated elderly Chinese. Int J Geriatr Psychiatry. 2003; 18(7):609-616.

19. Ma YC, Zuo L, Chen JH, et al. Modified glomerular filtration rate estimating equation for Chinese patients with chronic kidney disease. J Am Soc Nephrol. 2006;17(10):2937-2944.

20. Peters R, Beckett N, Fagard R, et al. Increased pulse pressure linked to dementia: further results from the hypertension in the very elderly trial - HYVET. J Hypertens. 2013;31(9):1868-1875.

21. Wang F, Zhang L, Liu L, Wang H. Level of kidney function correlates with cognitive decline. Am J Nephrol. 2010;32(2):117-121.

22. Kurella M, Chertow GM, Fried LF, et al. Chronic kidney disease and cognitive impairment in the elderly: the health, aging, and body composition study. J Am Soc Nephrol. 2005;16(7):2127-2133.
23. Khatri M, Nickolas T, Moon YP, et al. CKD associates with cognitive decline. J Am Soc Nephrol. 2009;20(11):2427-2432.

24. Slinin Y, Paudel ML, Ishani A, et al. Kidney function and cognitive performance and decline in older men. J Am Geriatr Soc. 2008;56(11): 2082-2088.

25. Mogi M, Horiuchi M. Clinical interaction between brain and kidney in small vessel disease. Cardiol Res Prac. 2011;2011:306189.

26. O'Rourke MF, Safar ME. Relationship between aortic stiffening and microvascular disease in brain and kidney: cause and logic of therapy. Hypertension. 2005;46(1):200-204.

27. Murray AM. The brain and the kidney connection: a model of accelerated vascular cognitive impairment. Neurology. 2009;73(12):916-917.

28. Atti AR, Palmer K, Volpato S, Zuliani G, Winblad B, Fratiglioni L. Anaemia increases the risk of dementia in cognitively intact elderly. Neurobiol Aging. 2006;27(2):278-284.

29. Seshadri S, Beiser A, Selhub J, et al. Plasma homocysteine as a risk factor for dementia and Alzheimer's disease. N Engl J Med. 2002; 346(7):476-483.

30. Zou G. A modified Poisson regression approach to prospective studies with binary data. Am J Epidemiol. 2004;159(7):702-706.
Clinical Interventions in Aging

\section{Publish your work in this journal}

Clinical Interventions in Aging is an international, peer-reviewed journal focusing on evidence-based reports on the value or lack thereof of treatments intended to prevent or delay the onset of maladaptive correlates of aging in human beings. This journal is indexed on PubMed Central, MedLine,

\section{Dovepress}

CAS, Scopus and the Elsevier Bibliographic databases. The manuscript management system is completely online and includes a very quick and fair peer-review system, which is all easy to use. Visit http://www.dovepress. com/testimonials.php to read real quotes from published authors. 